\title{
Homogeneous functional self-assembled monolayers: Faradaic impedance baseline signal drift suppression for high-sensitivity immunosensing of C- reactive protein
}

\author{
Prosper Kanyong, Jason J. Davis* \\ Department of Chemistry, University of Oxford, South Parks Road, Oxford, UK \\ "Corresponding author: jason.davis@chem.ox.ac.uk, Tel.: +44 (0)1865 275914
}

\begin{abstract}
The limit of detection (LOD) of affinity sensors based on alkanethiol self-assembled monolayers (SAMs) systems can be improved by either signal amplification and/or noise reduction. The latter includes baseline signal drift arising, in part, from, monolayer imperfections (and variations in this through repeats) as well as electrical noise of both the sensor and transducer. In reagentless "label free" assays signal drift carries with it the possibility of assay false-positive results (if signal drift is positive) or false-negatives (if specific signal is swamped by downward drift). Faradaic electrochemical impedance spectroscopy (FEIS) sensors based on SAM interfaces have been shown to exhibit considerable baseline signal drift; which consequently affects the LOD of such sensors. This study reports on the development of a simple two-step pre-treatment method for generating functional SAMs of 11-mercaptoundecanoic acid (MUA) on Au with a highly suppressed baseline signal drift. For electrochemical characterisation of the SAMs, the charge-transfer resistance $\left(R_{\mathrm{ct}}\right)$, defect presence (pinhole radius and separation), capacitance, and dielectric constant were evaluated. The interface pre-treatment generates films of substantially improved homogeneity that are
\end{abstract}


subsequently functionalised with anti-human C-reactive protein. CRP, an acute-phase protein, is subsequently detectable down to femtomolar levels without any amplification; this is a 2-3 order of magnitude lower detection limit than that typical accessible for analyses of this type. The developed protocols thus present a convenient and a general route to suppress baseline signal drifts and improve the limits of detection of affinity sensors based on faradaic impedance.

\section{Graphical abstract}

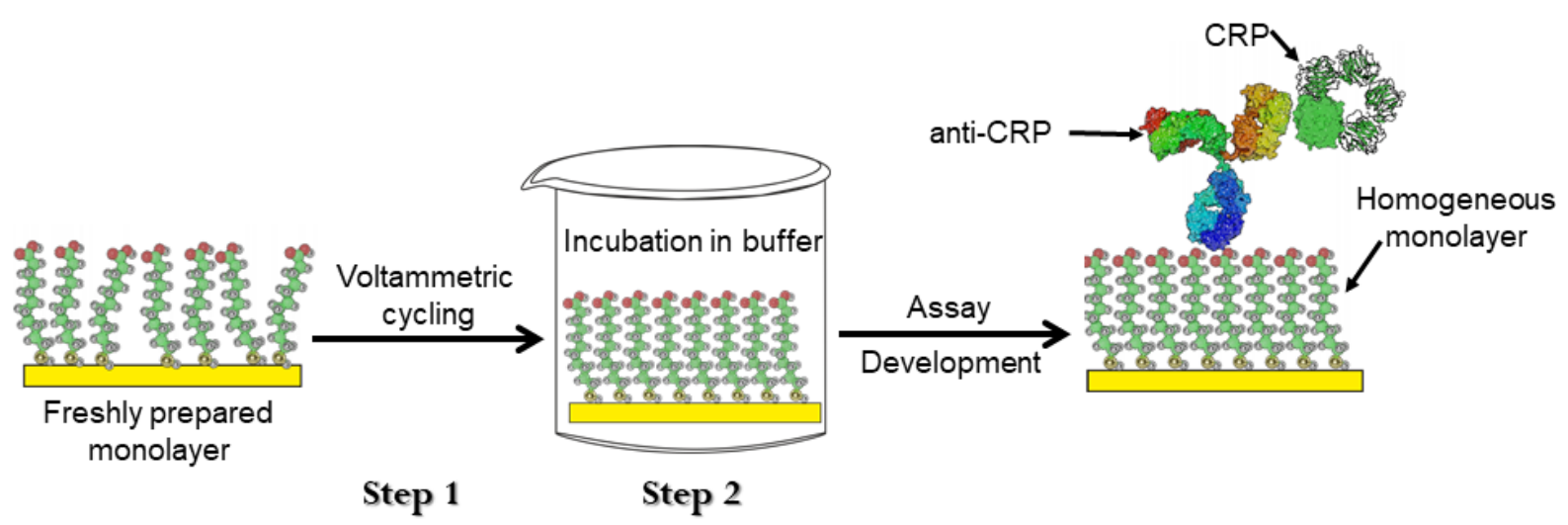

Schematic representation of a two-step pre-treatment method (voltammetric cycling, followed by incubation in buffer) for the generation of homogenous functional monolayers on gold for assay development. 


\section{Highlights}

- Mercaptoundecanoic acid (11-MUA) was self-assembled on gold from ethanolic solution. Faradaic EIS baseline drifts of the monolayers were evaluated in detail.

- A two-step protocol involving voltammetric cycling in buffered $\left[\mathrm{Fe}(\mathrm{CN})_{6}\right]^{3 / 4-}$ and immersion in PBS was developed to quench EIS baseline drifts and generate signals of unprecedented stability.

- A model is presented where changes are fully consistent with the underlying SAM becoming more homogeneous, with pinhole defects shrinking.

- A subsequently derived C-reactive protein assay has a remarkably high level of sensitivity, some two orders of magnitude greater than that which is typical for sensors of this type. 


\section{Introduction}

The design of label-free affinity-based sensors as alternative methods to traditional enzymelinked immunosorbent assays (ELISA) has been central to recent developments of potentially much improved diagnostic capability[1,2]. Among the various types of immunosensors, impedimetric immunosensors possess numerous attractive characteristics associated with the use of electrochemical transduction; they are easy to use, highly sensitive and entirely label free. Electroanalytical techniques, more generally, offer the possibility of achieving miniaturisation and integration of such capability into multi-array and/or microprocessorcontrolled diagnostic tools with remote control capabilities[1-3]; thus, making them portable, simple to operate, reliable and relatively inexpensive to manufacture. These favourable aspects offer much to the demands of point-of-care biomedical diagnostics[3,4].

Electrochemical impedance spectroscopy (EIS) is an a.c. method that describes the response of an electrochemical cell to a small amplitude sinusoidal voltage signal as a function of frequency. By measuring the differences in amplitude and phase angle of potential and current sinusoids over a range of frequency, the electrical properties of the electrode/electrolyte interface can mapped out to report on a number of time-dependent physical processes $[5,6]$. In a typical Faradaic impedance measurement, the interface can be characterised by the solution resistance $\left(R_{\mathrm{S}}\right)$, double-layer capacitance $\left(C_{\mathrm{dl}}\right)$ and charge-transfer resistance $\left(R_{\mathrm{ct}}\right)$. These parameters $\left(R_{\mathrm{s}}, \mathrm{C}_{\mathrm{dl}}\right.$ and $\left.R_{\mathrm{ct}}\right)$ are normally modelled and quantified using a Randles' equivalent circuit. Based on the nature of the transducing signal, impedimetric sensors can be classified as capacitive, where the surface of the electrode is covered by a dielectric layer and there is no resolved faradaic activity, or Faradaic, where the electrode confined film is either partial or conductive such that a solution phase redox probe is able to exchange electrons with the electrode[3]. In the latter case, (which is, by some margin, the most popular) the measured 
parameter is almost always the charge-transfer resistance $\left(R_{\mathrm{ct}}\right)$ and immunorecognition typically results in a steric/electrostatic increase in this[2,3].

Numerous electrode assemblies including oxide and metal oxide-based electrodes functionalised with silane, entrapped and/or covalently linked biomolecules on either conductive or electropolymerised non-conductive films-modified electrodes and gold (Au)covered alkanethiol-based self-assembled monolayers (SAMs) have been developed for impedimetric applications[1-3]. SAMs, in particular, provide a uniquely simple and convenient means of generating a functional interface but can present not insignificant EIS assay issues[7].It is known, for example, that SAMs generated under standard conditions are defective[8,9]. The subsequent effect of temperature on these defects has also been resolved by scanning tunnelling microscopy $(\mathrm{STM})[10,11]$ where a decrease in the number of islands is observed on warming (but the area of each island increases); this is an Ostwald ripening process, in which larger islands grow at the expense of smaller gold islands[12,13]. The microroughness (in the range of 1-10 nm) of SAM is also a key reporter of quality and structure[14]. In addition to monolayers containing defective pinhole sites, films may also include collapsed sites (locally thin regions of the monolayer resulting from the large orientated angle of thiol molecules near defects or domain boundaries). It has been shown that the electron transfer rate at pinholes is some 2 orders of magnitude greater than that at collapsed sites, where tunnelling is dominant [15]. The access of ions from solution to the underlying gold has also been evaluated by EIS with a view to noting pinhole presence and reporting on SAM quality. When compared to short chain alkanethiol SAMs, there has been, for example, a noted reduction in ion permeability for longer chains, attributed to predictably increased chain-chain interactions (as film crystallinity increases)[16-19]. It has been accepted, then, for some time 
now, that natively prepared monolayer have a variable number of defect sites of broadly understood character.

Once prepared, such films are not static. Using a combination of electrochemical and microscopic techniques, it has been previously shown, for example, that voltammetric cycling induces a film reorganisation in which the alkyl chains become more perpendicular to the electrode[20] markedly increasing their redox probe blocking properties[21,22]. It has been suggested that the imposition of a voltage can induce a torque on the thiolate headgroup, driving SAM structural change[19]. During incubation in solution, it is also known that SAMs are dynamic, especially when "young". Flynn et. al., studied the long-term stability of undecanethiol SAMs on $\mathrm{Au}$ during storage in phosphate-buffered saline (PBS) and calf serum[23] over a 35-day period. The advancing contact angles were observed to drop from $\sim 100^{\circ}$ to $>50^{\circ}$ after 21 days of storage in PBS and current densities obtained from cyclic voltammetry steadily increase. In a study of octadecanethiol (ODT) SAMs on gold by Faradaic EIS, Diao et al. have noted changes in fractional coverage $(\theta)$ as a function of incubation time (from $5 \mathrm{~s}$ to $24 \mathrm{~h}$ )[17]. The observations within this work were attributed to a gradual degradation of the monolayers via slow removal of the alkanethiol molecules from the Au surface. Further contact angle, cyclic voltammetry, FT-IR and XPS experiments reported in other work are additionally consistent with a progressive loss of alkanethiol molecules most likely via the oxidation of the thiolate headgroup[24,25]. The picture is, then, one of SAMs being inherently dynamic and defective where defects and film loss change over time in a manner that has significant dependency on imposed surface potential. The impact of this on sensor performance and baseline stability has been noted [26-28]. In any derived sensor, the limit of detection (LOD) is determined by the signal-to-noise ratio[26], the latter including (non-target induced) baseline drift as well as the electrical noise of both the sensor and 
transducer[26]. Consequently, the LOD of such sensors can be increased by either signal amplification and/or noise reduction. In this work we have sought to map out the FEIS baseline signal drift associated with SAMs of 11-mercaptoundecanoic acid (MUA) and quenched this through the application of a two-step protocol that utilises both an imposed cycling field and a solution phase incubation. The subsequent receptor modification of these films then enabled the generation of target specific sensors with a remarkably low detection limit.

\section{Experimental Section}

\subsection{Apparatus and reagents}

All chemical reagents used throughout this study were of analytical grade. 11mercaptoundecanoic acid (MUA), ethanol (99\%), phosphate buffered saline (PBS) tablets and potassium hexacyanoferrate (II) trihydrate ( $\geq 99.95 \%)$ were purchased from Sigma-Aldrich and used without any further purification. Potassium hexacyanoferrate (III) $(98+\%)$ was purchased from Lancaster Synthesis. Electrochemical experiments were conducted using PGSTAT204 Potentiostat/Galvanostat Autolab with FRA32M module (Metrohm-Autolab, The Netherlands) with Nova 2.1 Software for experimental control and data acquisition. Electrochemical impedance spectroscopy (EIS) in $1.0 \mathrm{mM}\left[\mathrm{Fe}(\mathrm{CN})_{6}\right]^{3 / 4-}$ redox couple was carried out at open circuit within the frequency range of $100 \mathrm{kHz}-0.5 \mathrm{~Hz}$ at an applied potential of $0.22 \mathrm{~V}$. Electrochemical measurements were performed using a three-electrode system where gold ( $\mathrm{Au})$ disk electrode (1.6 mm diameter, $\left.\mathrm{BASi}{ }^{\circledR} \mathrm{MF}-2014\right)$ served as the working electrode, $\mathrm{Pt}$ wire as counter electrode and $\mathrm{Ag} / \mathrm{AgCl}(3 \mathrm{M} \mathrm{NaCl})$ as reference electrode. The $\mathrm{Au}$ disk electrodes were purchased from $\mathrm{BASi}{ }^{\circledR}, \mathrm{USA}$. Alumina slurry of $1.0 \mu \mathrm{m}, 0.3 \mu \mathrm{m}$ and $0.05 \mu \mathrm{m}$ were purchased from Buehler, USA. Electrochemical measurements were carried out in 10.0 
$\mathrm{mM}$ PBS ( $\mathrm{pH} 7.4)$ in ultrapure water with resistivity of $18.2 \mathrm{M} \Omega \mathrm{cm}$. Ultrapure water was obtained from Milli-Q Type 1 Ultrapure Water system, Merck Millipore.

\subsection{Gold electrode cleaning}

Prior to self-assembly, the electrodes were mechanically polished with $1.0 \mu \mathrm{m}$ alumina slurry for $3.0 \mathrm{~min}$ and rinsed in distilled $\mathrm{H}_{2} \mathrm{O}$. The mechanical polishing was repeated using $0.3 \mu \mathrm{m}$ and $0.05 \mu \mathrm{m}$ alumina slurry, respectively. Thereafter, the electrodes were ultrasonicated in ethanol for $10.0 \mathrm{~min}$ to remove the alumina particles. This was then followed by immersion in piranha $\left(3: 1, \mathrm{H}_{2} \mathrm{SO}_{4}: \mathrm{H}_{2} \mathrm{O}_{2}\right)$ for $10.0 \mathrm{~min}$, rinsing in ultrapure water dried and drying under a gentle stream of $\mathrm{N}_{2}$. The electrodes were then electrochemically cycled from -0.3 to $1.6 \mathrm{~V}$ in $0.5 \mathrm{M} \mathrm{H}_{2} \mathrm{SO}_{4}$ at $0.1 \mathrm{~V} . \mathrm{s}^{-1}$ until stable voltammetric scans were obtained.

\subsection{MUA Monolayer preparation}

Immediately after cleaning, gold electrodes were dried with a gentle stream of $\mathrm{N}_{2}$ and immersed in $0.1 \mathrm{mM}$ ethanolic solution of MUA. After $18 \mathrm{hr}$ of SAM deposition, the electrodes were rinsed with ethanol, dried under a stream of $\mathrm{N}_{2}$, and Faradaic impedance spectra acquired in buffered $1.0 \mathrm{mM}\left[\mathrm{Fe}(\mathrm{CN})_{6}\right]^{3 / 4-}(10.0 \mathrm{mM}$ PBS, pH 7.4). The solution-phase Faradaic electrochemical impedance spectroscopy (FEIS) baseline drift of the monolayers was monitored as follows: after collecting the initial impedance scan, the SAM covered electrode was incubated in PBS (10.0 mM, pH 7.4) and FEIS scans were collected every $1 \mathrm{hr}$ for a period of up to 2 weeks. The temporal changes in $R_{\mathrm{ct}}$ were noted. The capacitance, dielectric constant and charge-transfer resistance $\left(R_{\mathrm{ct}}\right)$ of the monolayers were obtained by fitting the experimental 
data to an equivalent circuit consisting of the monolayer resistance parallel to the monolayer capacitance, in series with a solution resistance.

\subsection{Post-treatment of MUA monolayers}

The freshly prepared MUA SAMs have poorly resolved $R_{\mathrm{ct}}$ values and exhibited high FEIS temporal baseline signal drifts. The baseline drifts between any two consecutive impedance scans at $1 \mathrm{hr}$ time interval had coefficient of variation of over $15.0 \%$. Protocols were investigated to reduce the film impedance and increase temporal stability. In any sensor, the LOD is determined by the signal-to-noise ratio[26], the latter including (non-target induced) baseline drift as well as the electrical noise of both the sensor and transducer[26]. Consequently, the LOD of such sensors can be increased by either signal amplification and/or noise reduction. The aim for this study was to increase LOD via baseline noise reduction.

\subsubsection{Storage in buffer and buffered $\left[\mathrm{Fe}(\mathrm{CN})_{6}\right]^{3 / 4-}$}

The MUA monolayers were incubated in PBS (10.0 mM, pH 7.4) and buffered $1.0 \mathrm{mM}$ $\left[\mathrm{Fe}(\mathrm{CN})_{6}\right]^{3 / 4-}$ and Faradaic EIS were measured every $1 \mathrm{hr}$ up to 2 weeks. FEIS baseline signal drifts during the incubation were monitored with respect to time ( $R_{\mathrm{ct}} \mathrm{vs}$ time).

\subsubsection{Voltammetric cycling in buffer and buffered $[\mathrm{Fe}(\mathrm{CN}) 6]^{3 / 4-}$}

The monolayers were repeatedly cycled in PBS $(10.0 \mathrm{mM}$, pH 7.4) from -0.3 to $+0.6 \mathrm{~V}$ vs $\mathrm{Ag} / \mathrm{AgCl}$ at a scan rate of $0.1 \mathrm{~V} \cdot \mathrm{s}^{-1}$, for 150 cycles. Different cycle numbers ranging from 5 to 
500 were examined and 150 cycles found to be adequate in obtaining the baseline stability reported in this study. Cycles beyond 150 did not significantly change the properties of the SAM. After the cycling, the electrode was rinsed in PBS, dried in $\mathrm{N}_{2}$ and immersed in $1.0 \mathrm{mM}$ $\left[\mathrm{Fe}(\mathrm{CN})_{6}\right]^{3 / 4-}$ to acquire Faradaic EIS. Thereafter, the electrode was incubated in PBS for $1 \mathrm{hr}$ and FEIS acquired; this was repeated for several hrs and changes in $R_{\mathrm{ct}}$ monitored. This pretreatment procedure was also repeated in buffered $1.0 \mathrm{mM}\left[\mathrm{Fe}(\mathrm{CN})_{6}\right]^{3 / 4-}$. The monolayers were deemed stable when the coefficient of variation between $R_{\mathrm{ct}}$ values for consecutive measurements of up to $6 \mathrm{hr}$ was $\leq 1.0 \%$. All SAMs were stored in aluminium foil covered glass vials at room temperature in the dark throughout the experiments.

\subsection{Analytical application of the MUA monolayers}

For CRP assays, the carboxyl groups of the two-step pre-treated MUA monolayers were activated via immersion in aqueous solution of $0.4 \mathrm{M} N$-(3-(dimethylamino)propyl)- $N$ 'ethylcarbodiimide (EDC)/0.1 M N-hydroxysuccinimide (NHS) for $1 \mathrm{hr}$. Thereafter, the sensor was rinsed with PBS and incubated in $0.2 \mathrm{mg} / \mathrm{mL}$ anti-CRP in PBS (pH 7.4) for $1 \mathrm{hr}$. Finally, an incubation in $1.0 \%$ BSA (bovine serum albumin) solution for $30 \mathrm{~min}$ was performed. Calibration curves were obtained by immersion in PBS (pH 7.4) containing increasing specific concentrations of CRP (see Fig 4). Incubation times were 30 min for each CRP concentration and analysis performed after gently rinsing electrodes with PBS. 


\section{Results and discussion}

\subsection{Characterisation of freshly prepared MUA monolayers}

Observed changes in the electron transfer characteristics of a solution phase redox couple can provide useful information on the structural of an underlying electrode-confined film[29]. Using the $\left[\mathrm{Fe}(\mathrm{CN})_{6}\right]^{3 / 4-}$ redox couple in conjunction with cyclic voltammetry, the blocking properties of the monolayers are, for example, readily evaluated. The voltammetric profiles for freshly prepared MUA SAM-covered and bare Au are illustrated in Supplementary Information Fig. S1. It is evident that the as-prepared MUA SAM presents a high barrier to electron transfer as expected[30-32]. No apparent changes in the monolayer structure such as desorption or oxidation are noticed in the potential range employed. It is also noteworthy that the voltammogram shapes are typical of microelectrodes, an observation consistent with (limited) electron transfer being confined to defective sites[29,33,34]. The observed Nyquist data in Fig. S1B agree qualitatively well with the voltammetric profiles in Fig. S1A (with the electrontransfer resistance $\left(R_{\mathrm{ct}}\right)$ of the bare Au increased from $1.2 \mathrm{k} \Omega . \mathrm{cm}^{2}$ to $5.4 \mathrm{M} \Omega . \mathrm{cm}^{2}$; note that this is significantly higher than that afforded after treatment - see Fig. 1A). Importantly, these asprepared films have an impedance that is not only high (a feature we ascribe directly to the absence of significant pinhole defects[35]) but is also thereafter, unresponsive.

\subsection{Characterisation of freshly prepared MUA monolayers as a function of storage in buffer and buffered $\left[\mathrm{Fe}(\mathrm{CN})_{6}\right]^{3 / 4-}$}

Despite the volume of research on SAMs as (often assumed) biocompatible receptor supporting interfaces, relatively very little is known on solution-phase temporal FEIS baseline signal drifts or their subsequent suitability for biosensing applications[23]. To address this, a protocol was 
developed for generating homogenous functional monolayers. As noted above the incubation of SAMs in solution is known to promote dynamic change and plays a vital role in the formation of a stable film[23,24]. To map out the signal drift during storage in solution, the SAMs were firstly incubated in PBS and buffered $\left[\mathrm{Fe}(\mathrm{CN})_{6}\right]^{3 / 4-}$ and their baseline signal drifts monitored via FEIS. In general, during incubation in buffer (Fig. S2) or buffered $\left[\mathrm{Fe}(\mathrm{CN})_{6}\right]^{3 / 4}$, film $R_{\mathrm{ct}}$ is observed to decrease consistently such that FEIS baseline stabilities of $\leq 1 \%$ could not be obtained. This decrease is partly attributed to a reorganisation process of the SAM in which the total amount of exposed underlying $\mathrm{Au}$ (indicated by $R_{\mathrm{ct}}$ ) is largely constant[35] (and see below), but the film is both dynamic and populated by large pinhole defects through which the redox probe can access [34,36-38]. Dijksma et. al., observed similar continuous drift in FEIS baseline signal during storage of MUA monolayers in buffered $\left[\mathrm{Fe}(\mathrm{CN})_{6}\right]^{3 / 4}[39]$ and assigned this instead to partial film loss, as previously observed elsewhere[33,40]. The fractional surface coverage $(\theta)$ data (Section 3.4) generated herein does not support this. These significant FEIS baseline signal drifts meant that the monolayers, at this stage, were deemed unsuitable for subsequent sensing applications.

\subsection{Characterisation of voltammetrically cycled MUA monolayers as a function of storage in buffer and buffered $[\mathrm{Fe}(\mathrm{CN}) 6]^{3 / 4-}$}

Prior to storage in PBS, the SAMs were voltammetrically cycled in buffered $\left[\mathrm{Fe}(\mathrm{CN})_{6}\right]^{3 / 4}$. During the voltammetric cycling, there is a progressive change in film impedance and, thereafter, the Nyquist plots exhibit a more complete spectrum that includes (Fig. 1A) the Warburg low frequency region, an indication of an increase in presence of pinholes[17]. These observations are consistent with the occurrence of a reorganisation process in which significant pinhole defects are generated but the total amount of exposed $\mathrm{Au}$ (indicated by $R_{\mathrm{ct}}$ ) 
is largely constant by virtue of a concurrent decrease in the surface coverage of collapsed sites. At this stage, interfaces have a $R_{\mathrm{ct}}$ value that is typically $40 \%$ lower than "as prepared" films but still don't have an acceptable baseline stability. This is subsequently generated by prolonged ( $>12$ hours) incubation in aqueous solution. During this process $R_{\mathrm{ct}}$ falls by typically another $80 \%$ (see Fig. 1B) but, more importantly, is exceedingly stable (repeated impedance quantifications of $R_{\mathrm{ct}} \leq 1 \%$ ) for 2 weeks. Voltammetric cycling alone does not generate this stability. Aqueous incubation alone has little impact on the initially, prohibitively high, $R_{\mathrm{ct}}$. Both steps are, thus, necessary. We have, then, a picture where the imposed cycling induces a film rearrangement, a generation of substantial pinhole defects (and a resolution redox probe diffusion that is not prior resolved) followed by a subsequent "solidification" to a more crystalline and homogeneous state on subsequent incubation. An illustration of the temporal FEIS baseline signal drift of the monolayers before and after repeated voltammetric cycling, and during several hrs of incubation in PBS is shown in Fig. 1B. 

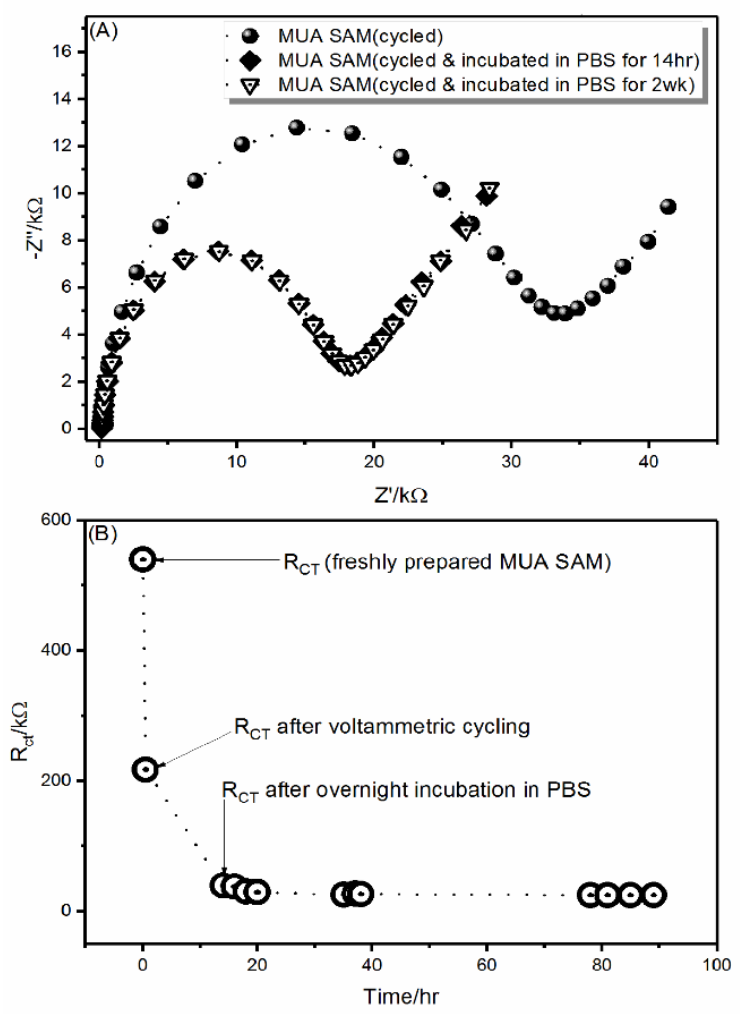

Fig. 1: (A) Nyquist plots for MUA SAM after voltammetric cycling in buffered $1.0 \mathrm{mM}$ $\left[\mathrm{Fe}(\mathrm{CN})_{6}\right]^{3 / 4-}$ followed by 14-hr and 2-week incubation in PBS (10.0 mM, pH 7.4); (B) An illustration of the temporal FEIS baseline drift in FEIS of voltammetrically cycled monolayers. The coefficient of variation (CV) between the $R_{\mathrm{ct}}$ values from $20 \mathrm{hr}$ to $90 \mathrm{hr}$ is $\leq 1 \%$.

This simple methodology of repeated voltammetric cycling in buffered $\left[\mathrm{Fe}(\mathrm{CN})_{6}\right]^{3 / 4-}$ (step one) and overnight incubation in buffer (step two), then, generates films of an unprecedented faradaic impedance baseline stability. 


\subsection{Electrochemical characterisation of defects in MUA monolayers}

As noted above the major defect types present in alkanethiol monolayers are those presented by pinholes and collapsed-sites (locally thin regions of the monolayer resulting from high molecular tilt)[41,42]. Molecules and/or ions from the electrolyte can reach the underlying electrode surface via the pinholes but they can also approach the electrode surface at a distance shorter than the full thickness of the monolayer at the collapsed sites[41,43,44]. The resolved $R_{\text {ct }}$ sheds light on these defects[41]. Since the rate of electron tunnelling decreases exponentially with increase of the barrier thickness, the electron transfer rate at defects will be much larger than at ordered SAM-covered domains[21,22,43,45]. To evaluate the quality of the two-step treated MUA monolayers, a quantitative examination of $\theta$, the pinhole size and separation, and collapsed sites was undertaken. The $\theta$ values for the monolayers were estimated using a model in which the pinholes are considered to be microelectrode in character (Eqn. 1);

$$
\theta=1-\frac{\left(\sigma_{\mathrm{w}}\right)}{\left(m-\sigma_{\mathrm{w}}\right)}
$$

where $\sigma_{\mathrm{w}}$ is the Warburg coefficient of bare Au electrode and $m$ is the slope of the linear interval observed in the high frequencies region of the $Z^{\prime}$ vs $\omega^{-1 / 2}$ function obtained at the MUA covered electrode. From Eqn. 1, values of 0.999 and 0.994 for freshly prepared MUA SAMs and twostep treated SAMs was obtained, respectively. The post-treated SAMs, then, have an almost identical total surface coverage to that presented by freshly prepared monolayers. Within this microarray model where the pinholes are assumed to be disk-shaped active regions with uniform radius and evenly distributed in the monolayer (Scheme 1C)[46], the radius of the pinholes $\left(\mathrm{r}_{\mathrm{a}}\right)$ in the monolayer and the separation distance between them $\left(\mathrm{r}_{\mathrm{b}}\right)$ was estimated. 


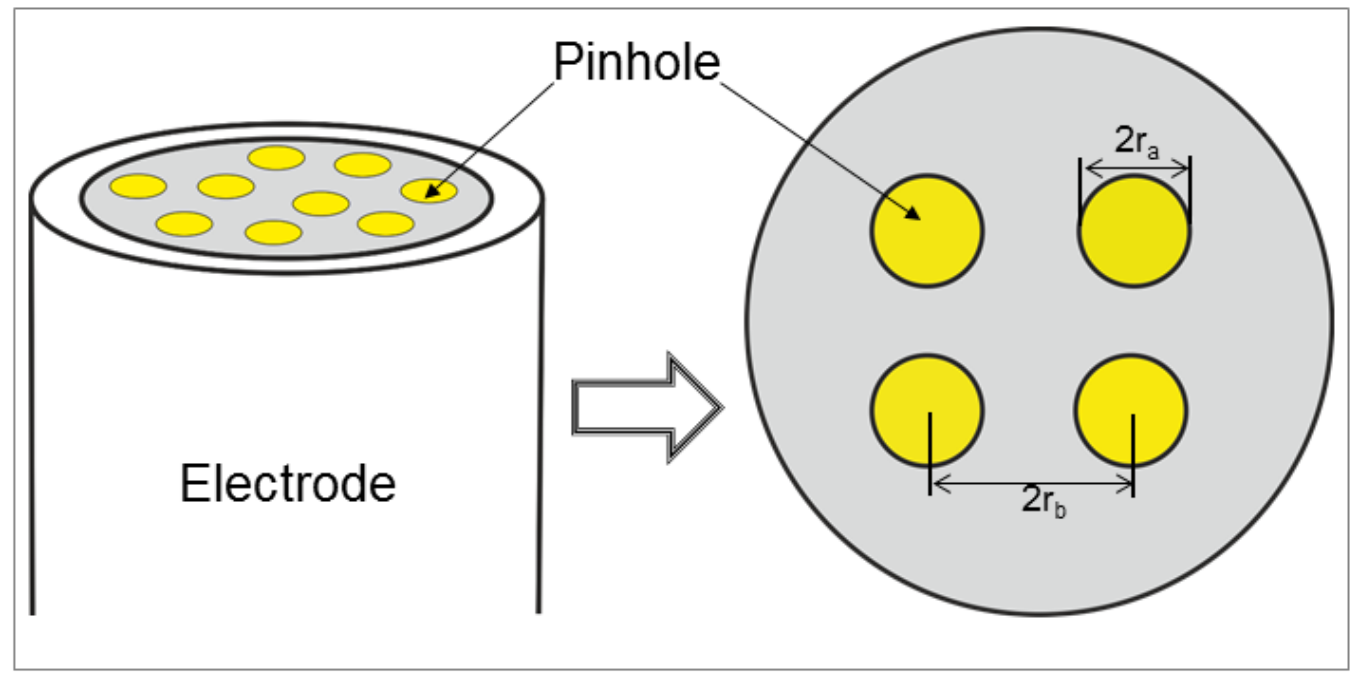

Scheme 1: Schematic representation of the radius of the pinhole $\left(r_{a}\right)$ and half the distance between the centres of adjacent pinholes $\left(\mathrm{r}_{\mathrm{b}}\right)$.

The fraction of the pinhole area $(1-\theta)$, is related to $r_{a}$ and $r_{b}$ by Eqn. 2:

$$
1-\theta=\frac{\left(\mathrm{r}_{\mathrm{a}}^{2}\right)}{\left(\mathrm{r}_{\mathrm{b}}^{2}\right)}
$$

but $r_{a}$ can be estimated from the frequency value corresponding to the turning point between the high and low frequency domain in the $Z^{\prime \prime}$ vs $\omega^{-1 / 2}$ plot $[43,47-49]$ in Fig. 2 . The $Z^{\prime \prime}$ vs $\omega^{-1 / 2}$ for the MUA SAM covered Au electrode shows a maximum (the turning point) at $0.5 \mathrm{~Hz}$ (Fig. 2A) and 58.7 Hz (Fig. 2B) for freshly prepared and two-step treated monolayers, respectively. 
Using this frequency values in conjunction with Eqn. 3:

$$
\omega=\frac{\mathrm{q}}{2}
$$

(where $\omega=2 \pi \mathrm{f}$, $\mathrm{f}$ is the impedance frequency and $q$ is the transition radial frequency), $q$ was determined, used in the expression in Eqn. 4:

$$
q=\frac{D}{0.36 \mathrm{r}_{\mathrm{a}}^{2}}
$$

(where $D$ is the diffusion coefficient for potassium hexacyanoferrate (III) (here $D=7.56 \times 10^{-}$ $\left.{ }^{6} \mathrm{~cm}^{2} \mathrm{~s}^{-1}\right)[50]$ and $\mathrm{r}_{\mathrm{a}}$ values of the freshly prepared and two-step treated MUA SAM were estimated to be $19.0 \mu \mathrm{m}( \pm 0.3 \mu \mathrm{m})$ and $1.7 \mu \mathrm{m}( \pm 0.4 \mu \mathrm{m})$, respectively. Clearly, the pinhole radius in the MUA monolayers decreases significantly through the two-step treatment (pinholes generated in the first step and homogenised in the second). Because the film $R_{\mathrm{ct}}$, and the total film coverage it reports, is largely constant through this second step, this must necessarily be associated with a decrease in the number of collapsed sites. The increase in film order will be associated with increased stabilising intermolecular interactions[21,22,58,34,5157] and it is this that we believe to be responsible for the high levels of baseline stability thereafter observed.

Based on the values of $r_{a}$ and $r_{b}$, and the induced changes in the area of exposed $\mathrm{Au}$, the total number of film-generated microelectrodes was estimated to be $\sim 2$ and $\sim 320$, before and after treatment. This data analysis suggests that the pre-treatment method leads to a reorganisation process that increases the pinhole density, reduces the number of collapsed sites and generates films of substantially pronounced homogeneity. 

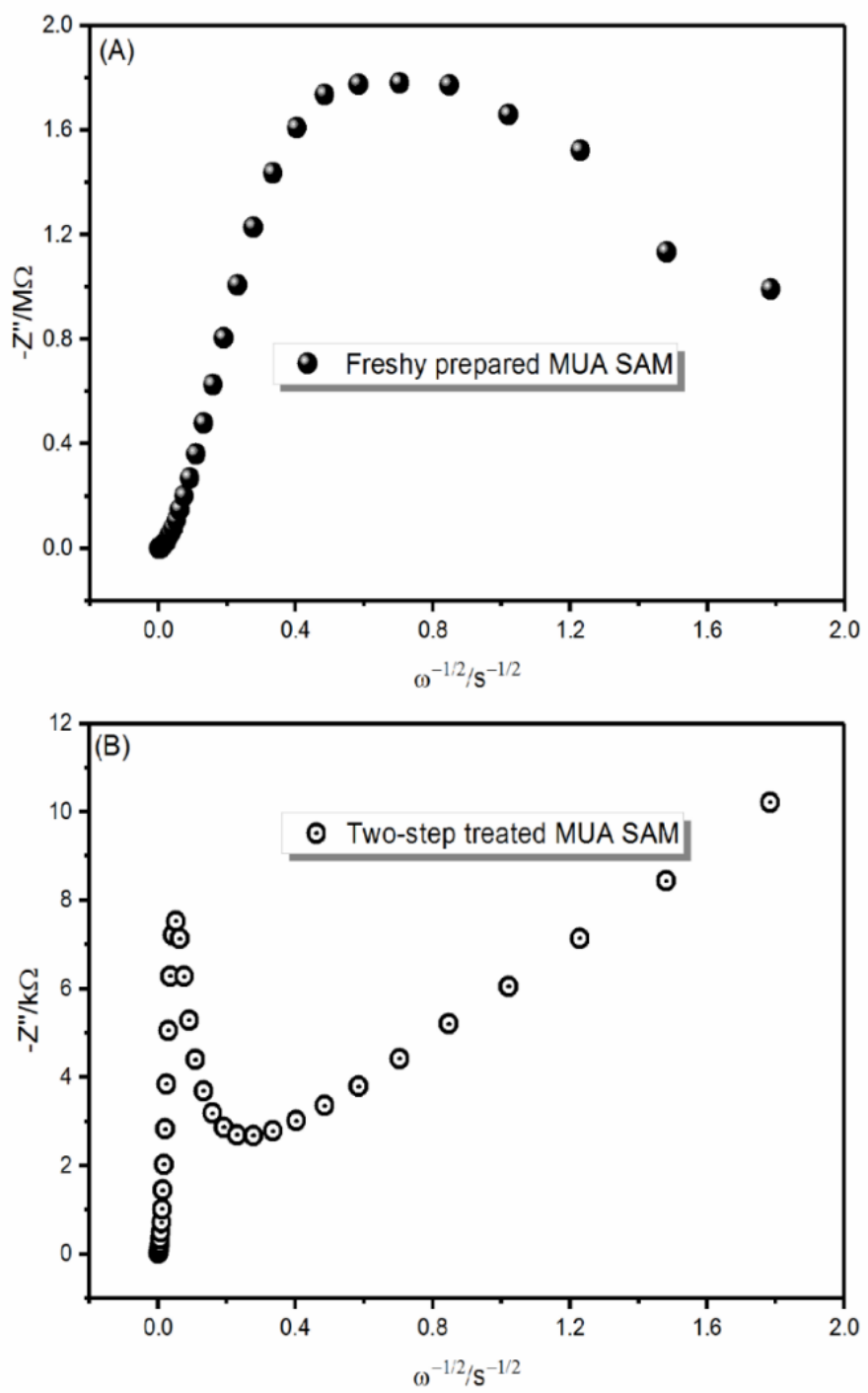

Fig. 2: Relationship of imaginary part of Faradaic impedance $\left(Z^{\prime \prime}\right)$ as a function of $\omega^{-1 / 2}$ for $\mathrm{Au}$ covered MUA SAM showing the maximum the turning point. The frequency values at the maximum turning point (used to calculate $\mathrm{r}_{\mathrm{a}}$ and $\mathrm{r}_{\mathrm{b}}$ ) increased from $0.5 \mathrm{~Hz}$ to $59 \mathrm{~Hz}$ for freshly prepared and two-step treated SAMs, respectively (directly reporting on film pinhole size changes. -see main text). 


\subsection{Characterisation of treated MUA monolayers: capacitance and dielectric properties}

To further analyse the quality of the treated MUA interfaces, capacitance and dielectric (Fig. S4) changes during incubation in PBS (10.0 mM, pH 7.4) were evaluated and illustrated in Fig. 3. Dielectric constant values were calculated using the expression in Fig. S4. The complex

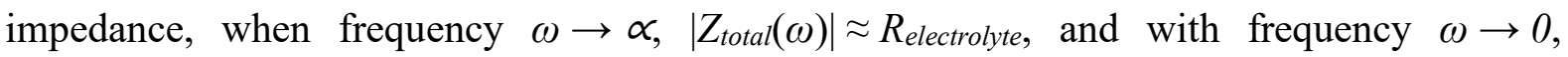
$\left|Z_{\text {total }}(\omega)\right| \approx 1 / \omega \cdot C_{S A M}[48,59]$. Variance of the capacitance with time was computed at a frequency of $1000 \mathrm{~Hz}$. The interfacial capacitance and dielectric constants agree with those obtained for well-packed ordered alkanethiol SAMs of minimal solvent penetration (grey shaded areas on Fig. 3)[40,60-62].

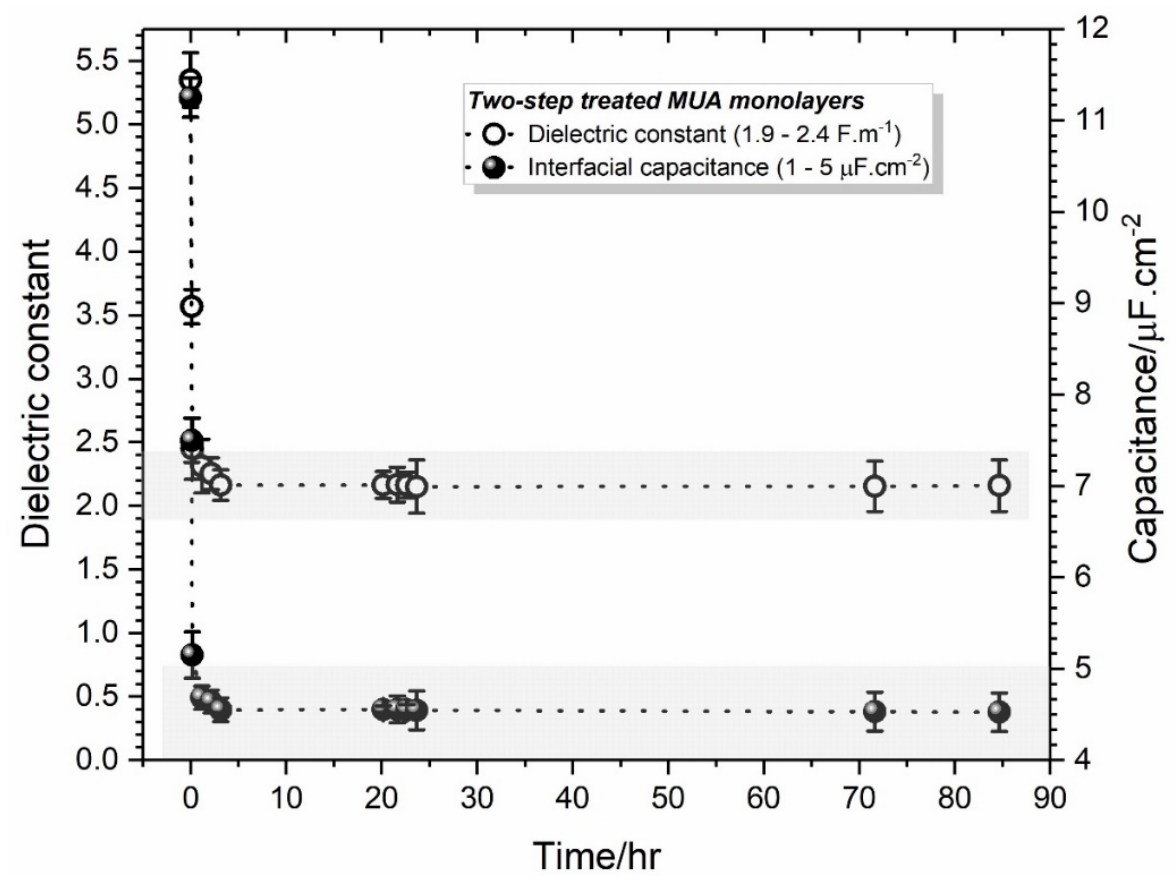

Fig. 3: Dielectric constants $(\varepsilon)$ and interfacial capacitances of two-step treated MUA SAMs during incubation in PBS (10.0 mM, pH 7.4). Errors bars are standard deviations of 6 repeat measurements 


\subsection{Analytical application of the MUA monolayers}

The MUA monolayers were subsequently applied to the development of an immunosensor for C-reactive protein (CRP) (Fig. 4) through the covalent immobilisation of monoclonal antiCRP. Fig. 4A gives an overview of the Nyquist resolved changes on anti-CRP antibody immobilisation, the blocking of any unoccupied surface with BSA (1.0\%) and the subsequent direct recognition of CRP in PBS (10.0 mM, pH 7.4). The corresponding CRP calibration curve is shown in Fig. 4B. A linear range for CRP was recorded from 200.0 to $5000.0 \mu \mathrm{g} / \mathrm{L}$ (1.75 to $44 \mathrm{nM}$ ) with a sensitivity of $2.0 \Omega$. $(\mathrm{mg} / \mathrm{L}) . \mathrm{cm}^{-2}$ and a calculated limit of detection (based on $3 \mathrm{x}$ the baseline noise) of $3.7 \mathrm{ng} / \mathrm{L}(32 \mathrm{fM})$. This low detection limit and/or wide linear range are notably better than previously reported for any prior SAM (including 11-MUA) based faradaic impedance sensor[63-68]. 

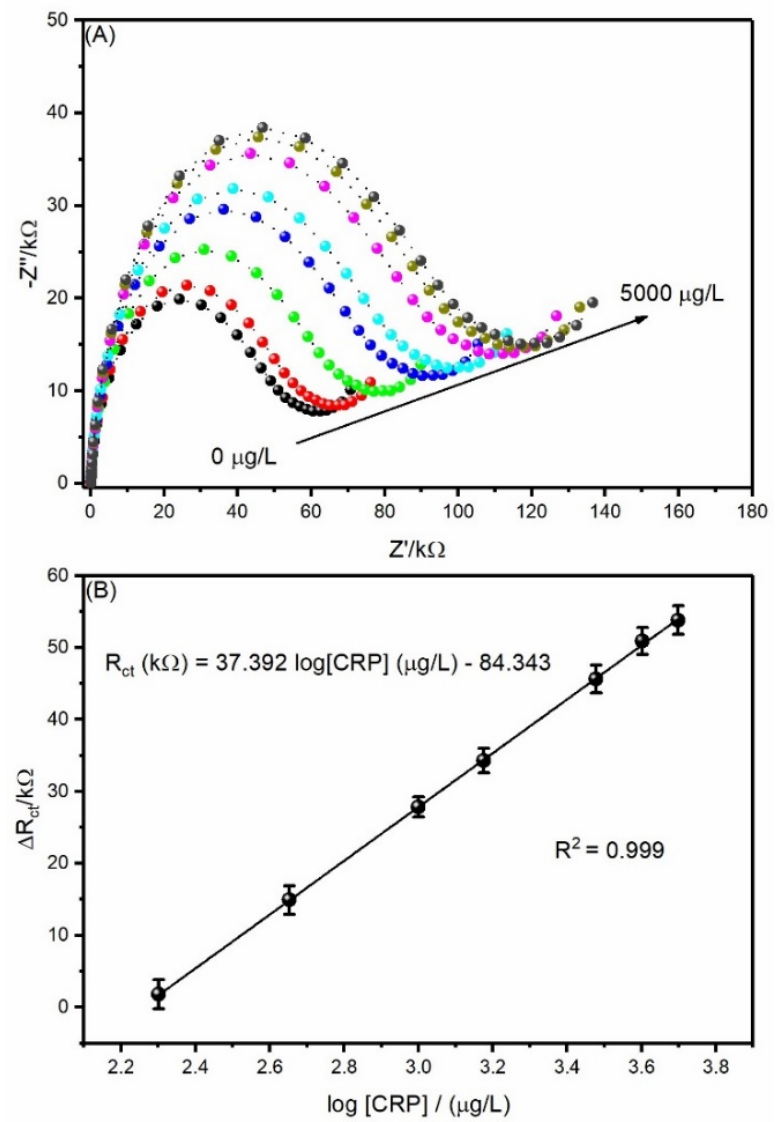

Fig. 4: (A) Nyquist plots for increasing concentrations of CRP and the (B) Corresponding calibration plot for CRP; grey shaded areas indicates the levels in human blood and their associated risk for developing cardiovascular diseases. The concentrations of CRP used are $200 \mu \mathrm{g} / \mathrm{L}, 450 \mu \mathrm{g} / \mathrm{L}, 1000 \mu \mathrm{g} / \mathrm{L}, 1500 \mu \mathrm{g} / \mathrm{L}, 3000 \mu \mathrm{g} / \mathrm{L}, 40000 \mu \mathrm{g} / \mathrm{L}$ and $5000 \mu \mathrm{g} / \mathrm{L}$. Errors bars represent one standard deviation across three independent measurements.

\section{Conclusions}

A simple two-step pre-treatment method, comprising an initial voltammetric cycling of freshly formed MUA SAMs in buffered $\left[\mathrm{Fe}(\mathrm{CN})_{6}\right]^{3 / 4-}$, then an overnight incubation in PBS, has been developed for the generation of homogenous films for which the associated impedance baseline drift, something that commonly "poisons" reagentless electroanalytical assays, is largely 
removed. Our model is that, as prepared films (on very clean gold substrates) present a prohibitively high and unresponsive $R_{\mathrm{ct}}$. A markedly lower and responsive $R_{\mathrm{ct}}$ is generatable by inducing pinhole defects by voltammetric cycling. This is then "crystallised" into the film by aqueous incubation (a process we believe to promote lateral intermolecular interactions). A quantitative examination of monolayer fractional coverage $(\theta)$, defects (pinhole radius and separation) resolves a decrease in film pinhole defects from $19.0 \mu \mathrm{m}$ to $1.7 \mu \mathrm{m}$ through this second step, indicating both a decrease in the number of collapsed film sites and an increase in film homogeneity. Resolved interfacial capacitance and dielectric constant fingerprints support this picture. The improvement in the baseline stability enabled the detection of C-reactive protein (CRP), a classical acute-phase protein with an LOD that is 2-3 orders of magnitude better than that previously reported for films of this type.

\section{Acknowledgments}

The authors gratefully acknowledge the financial support given to this work by Osler Diagnostics. 


\section{References}

[1] Q. Xu, J.J. Davis, The Diagnostic Utility of Electrochemical Impedance, Electroanalysis. 26 (2014) 1249-1258. https://doi.org/10.1002/elan.201400035.

[2] J.-G. Guan, Y.-Q. Miao, Q.-J. Zhang, Impedimetric biosensors, J. Biosci. Bioeng. 97 (2004) 219-226. https://doi.org/https://doi.org/10.1016/S1389-1723(04)70195-4.

[3] M.I. Prodromidis, Impedimetric immunosensors-A review, Electrochim. Acta. 55 (2010) 4227-4233. https://doi.org/https://doi.org/10.1016/j.electacta.2009.01.081.

[4] D.W. Kimmel, G. LeBlanc, M.E. Meschievitz, D.E. Cliffel, Electrochemical Sensors and Biosensors, Anal. Chem. 84 (2012) 685-707. https://doi.org/10.1021/ac202878q.

[5] B.-Y. Chang, S.-M. Park, Electrochemical Impedance Spectroscopy, Annu. Rev. Anal. Chem. 3 (2010) 207-229. https://doi.org/10.1146/annurev.anchem.012809.102211.

[6] F. Lisdat, D. Schäfer, The use of electrochemical impedance spectroscopy for biosensing, Anal. Bioanal. Chem. 391 (2008) 1555. https://doi.org/10.1007/s00216008-1970-7.

[7] D.D. Agonafer, E. Chainani, M.E. Oruc, K.S. Lee, M.A. Shannon, Study of Insulating Properties of Alkanethiol Self-Assembled Monolayers Formed Under Prolonged Incubation Using Electrochemical Impedance Spectroscopy, J. Nanotechnol. Eng. Med. 3 (2013) 31006-31008. http://dx.doi.org/10.1115/1.4007698.

[8] C. Vericat, M.E. Vela, R.C. Salvarezza, Self-assembled monolayers of alkanethiols on $\mathrm{Au}(111)$ : surface structures, defects and dynamics, Phys. Chem. Chem. Phys. 7 (2005) 3258-3268. https://doi.org/10.1039/B505903H.

[9] C. Vericat, M.E. Vela, G. Benitez, P. Carro, R.C. Salvarezza, Self-assembled monolayers of thiols and dithiols on gold: new challenges for a well-known system, Chem. Soc. Rev. 39 (2010) 1805-1834. https://doi.org/10.1039/B907301A.

[10] R. Yamada, H. Wano, K. Uosaki, Effect of Temperature on Structure of the SelfAssembled Monolayer of Decanethiol on $\mathrm{Au}(111)$ Surface, Langmuir. 16 (2000) 5523-5525. https://doi.org/10.1021/la991394e.

[11] G.E. Poirier, M.J. Tarlov, Molecular ordering and gold migration observed in butanethiol self-assembled monolayers using scanning tunneling microscopy, J. Phys. Chem. 99 (1995) 10966-10970. https://doi.org/10.1021/j100027a042.

[12] I.M. Lifshitz, V. V Slyozov, The kinetics of precipitation from supersaturated solid solutions, J. Phys. Chem. Solids. 19 (1961) 35-50.

https://doi.org/https://doi.org/10.1016/0022-3697(61)90054-3.

[13] T.K.N. Hoang, L. Deriemaeker, V.B. La, R. Finsy, Monitoring the Simultaneous Ostwald Ripening and Solubilization of Emulsions, Langmuir. 20 (2004) 8966-8969. https://doi.org/10.1021/la049184b.

[14] E.F. Douglass Jr., P.F. Driscoll, D. Liu, N.A. Burnham, C.R. Lambert, W.G. McGimpsey, Effect of Electrode Roughness On the Capacitive Behavior of SelfAssembled Monolayers, Anal. Chem. 80 (2008) 7670-7677. 
https://doi.org/10.1021/ac800521z.

[15] H.O. Finklea, S. Avery, M. Lynch, T. Furtsch, Blocking oriented monolayers of alkyl mercaptans on gold electrodes, Langmuir. 3 (1987) 409-413.

https://doi.org/10.1021/la00075a024.

[16] F. Schreiber, Structure and growth of self-assembling monolayers, Prog. Surf. Sci. 65 (2000) 151-257. https://doi.org/https://doi.org/10.1016/S0079-6816(00)00024-1.

[17] P. Diao, M. Guo, R. Tong, Characterization of defects in the formation process of selfassembled thiol monolayers by electrochemical impedance spectroscopy, $\mathrm{J}$.

Electroanal. Chem. 495 (2001) 98-105. https://doi.org/https://doi.org/10.1016/S00220728(00)00424-1.

[18] C. Gupta, M.A. Shannon, P.J.A. Kenis, Electronic Properties of a Monolayer-Electrolyte Interface Obtained from Mechanistic Impedance Analysis, J. Phys. Chem. C. 113 (2009) 9375-9391. https://doi.org/10.1021/jp900918u.

[19] H. Sahalov, B. O’Brien, K.J. Stebe, K. Hristova, P.C. Searson, Influence of Applied Potential on the Impedance of Alkanethiol SAMs, Langmuir. 23 (2007) 9681-9685. https://doi.org/10.1021/la701398u.

[20] S. Ye, Y. Sato, K. Uosaki, Redox-Induced Orientation Change of a Self-Assembled Monolayer of 11-Ferrocenyl-1-undecanethiol on a Gold Electrode Studied by in Situ FT-IRRAS, Langmuir. 13 (1997) 3157-3161. https://doi.org/10.1021/la9700432.

[21] V. Ganesh, V. Lakshminarayanan, Scanning Tunneling Microscopy, Fourier Transform Infrared Spectroscopy, and Electrochemical Characterization of 2Naphthalenethiol Self-Assembled Monolayers on the Au Surface: A Study of BridgeMediated Electron Transfer in Ru(NH3)62+|Ru(NH3)63+ Redox Re, J. Phys. Chem. B. 109 (2005) 16372-16381. https://doi.org/10.1021/jp052489u.

[22] V. Ganesh, R.R. Pandey, B.D. Malhotra, V. Lakshminarayanan, Electrochemical characterization of self-assembled monolayers (SAMs) of thiophenol and aminothiophenols on polycrystalline Au: Effects of potential cycling and mixed SAM formation, J. Electroanal. Chem. 619-620 (2008) 87-97. https://doi.org/https://doi.org/10.1016/j.jelechem.2008.03.015.

[23] N.T. Flynn, T.N.T. Tran, M.J. Cima, R. Langer, Long-Term Stability of SelfAssembled Monolayers in Biological Media, Langmuir. 19 (2003) 10909-10915. https://doi.org/10.1021/la035331e.

[24] G. Mani, D.M. Johnson, D. Marton, V.L. Dougherty, M.D. Feldman, D. Patel, A.A. Ayon, C.M. Agrawal, Stability of Self-Assembled Monolayers on Titanium and Gold, Langmuir. 24 (2008) 6774-6784. https://doi.org/10.1021/la8003646.

[25] N.T. Flynn, T.N.T. Tran, M.J. Cima, R. Langer, Long-Term Stability of SelfAssembled Monolayers in Biological Media, Langmuir. 19 (2003) 10909-10915. https://doi.org/10.1021/la035331e.

[26] M. Riepl, V.M. Mirsky, I. Novotny, V. Tvarozek, V. Rehacek, O.S. Wolfbeis, Optimization of capacitive affinity sensors: drift suppression and signal amplification, 
Anal. Chim. Acta. 392 (1999) 77-84. https://doi.org/https://doi.org/10.1016/S00032670(99)00195-6.

[27] A. Bogomolova, E. Komarova, K. Reber, T. Gerasimov, O. Yavuz, S. Bhatt, M. Aldissi, Challenges of Electrochemical Impedance Spectroscopy in Protein Biosensing, Anal. Chem. 81 (2009) 3944-3949. https://doi.org/10.1021/ac9002358.

[28] J. Lazar, C. Schnelting, E. Slavcheva, U. Schnakenberg, Hampering of the Stability of Gold Electrodes by Ferri-/Ferrocyanide Redox Couple Electrolytes during Electrochemical Impedance Spectroscopy, Anal. Chem. 88 (2016) 682-687. https://doi.org/10.1021/acs.analchem.5b02367.

[29] R.P. Janek, W.R. Fawcett, A. Ulman, Impedance Spectroscopy of Self-Assembled Monolayers on $\mathrm{Au}(111)$ : Sodium Ferrocyanide Charge Transfer at Modified Electrodes, Langmuir. 14 (1998) 3011-3018. https://doi.org/10.1021/la970980+.

[30] F. van Voorst Vader, Composition of the interface of carboxylate soap solutions, Trans. Faraday Soc. 57 (1961) 2263-2271. https://doi.org/10.1039/TF9615702263.

[31] E. Cooper, G.J. Leggett, Influence of Tail-Group Hydrogen Bonding on the Stabilities of Self-Assembled Monolayers of Alkylthiols on Gold, Langmuir. 15 (1999) 10241032. https://doi.org/10.1021/la980795b.

[32] C.D. Bain, G.M. Whitesides, A study by contact angle of the acid-base behavior of monolayers containing .omega.-mercaptocarboxylic acids adsorbed on gold: an example of reactive spreading, Langmuir. 5 (1989) 1370-1378. https://doi.org/10.1021/la00090a019.

[33] R.M. Wightman, Microvoltammetric electrodes, Anal. Chem. 53 (1981) 1125A1134A. https://doi.org/10.1021/ac00232a004.

[34] M.D. Porter, T.B. Bright, D.L. Allara, C.E.D. Chidsey, Spontaneously organized molecular assemblies. 4. Structural characterization of n-alkyl thiol monolayers on gold by optical ellipsometry, infrared spectroscopy, and electrochemistry, J. Am. Chem. Soc. 109 (1987) 3559-3568. https://doi.org/10.1021/ja00246a011.

[35] M. Dijksma, B.A. Boukamp, B. Kamp, W.P. van Bennekom, Effect of Hexacyanoferrate(II/III) on Self-Assembled Monolayers of Thioctic Acid and 11Mercaptoundecanoic Acid on Gold, Langmuir. 18 (2002) 3105-3112. https://doi.org/10.1021/la0108981.

[36] K. Shimazu, I. Yagi, Y. Sato, K. Uosaki, In situ and dynamic monitoring of the selfassembling and redox processes of a ferrocenylundecanethiol monolayer by electrochemical quartz crystal microbalance, Langmuir. 8 (1992) 1385-1387. https://doi.org/10.1021/la00041a023.

[37] S. Xu, S.J.N. Cruchon-Dupeyrat, J.C. Garno, G.-Y. Liu, G. Kane Jennings, T.-H. Yong, P.E. Laibinis, In situ studies of thiol self-assembly on gold from solution using atomic force microscopy, J. Chem. Phys. 108 (1998) 5002-5012. https://doi.org/10.1063/1.475908.

[38] D.S. Karpovich, G.J. Blanchard, Direct Measurement of the Adsorption Kinetics of 
Alkanethiolate Self-Assembled Monolayers on a Microcrystalline Gold Surface, Langmuir. 10 (1994) 3315-3322. https://doi.org/10.1021/la00021a066.

[39] M. Dijksma, B.A. Boukamp, B. Kamp, W.P. van Bennekom, Effect of Hexacyanoferrate(II/III) on Self-Assembled Monolayers of Thioctic Acid and 11Mercaptoundecanoic Acid on Gold, Langmuir. 18 (2002) 3105-3112. https://doi.org/10.1021/la0108981.

[40] M. Riepl, V.M. Mirsky, I. Novotny, V. Tvarozek, V. Rehacek, O.S. Wolfbeis, Optimization of capacitive affinity sensors: drift suppression and signal amplification, Anal. Chim. Acta. 392 (1999) 77-84. https://doi.org/https://doi.org/10.1016/S00032670(99)00195-6.

[41] J. Stettner, P. Frank, T. Griesser, G. Trimmel, R. Schennach, E. Gilli, A. Winkler, A Study on the Formation and Thermal Stability of 11-MUA SAMs on Au(111)/Mica and on Polycrystalline Gold Foils, Langmuir. 25 (2009) 1427-1433. https://doi.org/10.1021/la802534q.

[42] P. Diao, D. Jiang, X. Cui, D. Gu, R. Tong, B. Zhong, Studies of structural disorder of self-assembled thiol monolayers on gold by cyclic voltammetry and ac impedance, J. Electroanal. Chem. 464 (1999) 61-67. https://doi.org/https://doi.org/10.1016/S00220728(98)00470-7.

[43] R.A. Marcus, N. Sutin, Electron transfers in chemistry and biology, Biochim. Biophys. Acta - Rev. Bioenerg. 811 (1985) 265-322.

https://doi.org/https://doi.org/10.1016/0304-4173(85)90014-X.

[44] P. Diao, M. Guo, R. Tong, Characterization of defects in the formation process of selfassembled thiol monolayers by electrochemical impedance spectroscopy, J.

Electroanal. Chem. 495 (2001) 98-105. https://doi.org/https://doi.org/10.1016/S00220728(00)00424-1.

[45] Y. V Sukhinin, Phase transitions in self-assembled monolayers of alkanethiols containing the polar group, J. Exp. Theor. Phys. 87 (1998) 115-122. https://doi.org/10.1134/1.558645.

[46] H.O. Finklea, D.A. Snider, J. Fedyk, E. Sabatani, Y. Gafni, I. Rubinstein, Characterization of octadecanethiol-coated gold electrodes as microarray electrodes by cyclic voltammetry and ac impedance spectroscopy, Langmuir. 9 (1993) 3660-3667. https://doi.org/10.1021/la00036a050.

[47] C. Amatore, J.M. Savéant, D. Tessier, Charge transfer at partially blocked surfaces: A model for the case of microscopic active and inactive sites, J. Electroanal. Chem. Interfacial Electrochem. 147 (1983) 39-51. https://doi.org/https://doi.org/10.1016/S0022-0728(83)80055-2.

[48] S. Campuzano, M. Pedrero, C. Montemayor, E. Fatás, J.M. Pingarrón, Characterization of alkanethiol-self-assembled monolayers-modified gold electrodes by electrochemical impedance spectroscopy, J. Electroanal. Chem. 586 (2006) 112121. https://doi.org/https://doi.org/10.1016/j.jelechem.2005.09.007.

[49] K. Tokuda, T. Gueshi, H. Matsuda, Voltammetry at partially covered electrodes: Part 
III. Faradaic impedance measurements at model electrodes, J. Electroanal. Chem. Interfacial Electrochem. 102 (1979) 41-48.

https://doi.org/https://doi.org/10.1016/S0022-0728(79)80027-3.

[50] J.J. Gooding, V.G. Praig, E.A.H. Hall, Platinum-Catalyzed Enzyme Electrodes Immobilized on Gold Using Self-Assembled Layers, Anal. Chem. 70 (1998) 23962402. https://doi.org/10.1021/ac971035t.

[51] K.V.G.K. Murty, M. Venkataramanan, T. Pradeep, Self-assembled Monolayers of 1,4Benzenedimethanethiol on Polycrystalline Silver and Gold Films: An Investigation of Structure, Stability, Dynamics, and Reactivity, Langmuir. 14 (1998) 5446-5456. https://doi.org/10.1021/la980249i.

[52] L. Srisombat, A.C. Jamison, T.R. Lee, Stability: A key issue for self-assembled monolayers on gold as thin-film coatings and nanoparticle protectants, Colloids Surfaces A Physicochem. Eng. Asp. 390 (2011) 1-19. https://doi.org/https://doi.org/10.1016/j.colsurfa.2011.09.020.

[53] G.E. Poirier, M.J. Tarlov, H.E. Rushmeier, Two-Dimensional Liquid Phase and the px.sqroot.3 Phase of Alkanethiol Self-Assembled Monolayers on Au(111), Langmuir. 10 (1994) 3383-3386. https://doi.org/10.1021/la00022a004.

[54] R.S. Clegg, S.M. Reed, J.E. Hutchison, Self-Assembled Monolayers Stabilized by Three-Dimensional Networks of Hydrogen Bonds, J. Am. Chem. Soc. 120 (1998) 2486-2487. https://doi.org/10.1021/ja9800277.

[55] D.L. Allara, A.N. Parikh, E. Judge, The existence of structure progressions and wetting transitions in intermediately disordered monolayer alkyl chain assemblies, J. Chem. Phys. 100 (1994) 1761-1764. https://doi.org/10.1063/1.466604.

[56] J.C. Love, L.A. Estroff, J.K. Kriebel, R.G. Nuzzo, G.M. Whitesides, Self-Assembled Monolayers of Thiolates on Metals as a Form of Nanotechnology, Chem. Rev. 105 (2005) 1103-1170. https://doi.org/10.1021/cr0300789.

[57] D. Samanta, A. Sarkar, Immobilization of bio-macromolecules on self-assembled monolayers: methods and sensor applications, Chem. Soc. Rev. 40 (2011) 2567-2592. https://doi.org/10.1039/C0CS00056F.

[58] J.J. Calvente, G. López-Pérez, J.M. Jurado, R. Andreu, M. Molero, E. Roldán, Reorientation of Thiols during 2D Self-Assembly: Interplay between Steric and Energetic Factors, Langmuir. 26 (2010) 2914-2923. https://doi.org/10.1021/la902981n.

[59] M. Jalal Uddin, M. Khalid Hossain, M.I. Hossain, W. Qarony, S. Tayyaba, M.N.H. Mia, M.F. Pervez, S. Hossen, Modeling of self-assembled monolayers (SAMs) of Octadecanethiol and Hexadecanethiol on gold $(\mathrm{Au})$ and silver (Ag), Results Phys. 7 (2017) 2289-2295. https://doi.org/https://doi.org/10.1016/j.rinp.2017.06.055.

[60] N.N. and M.H. and H.S. and W. Knoll, Thermal Desorption Spectroscopy of Alkanethiol Self-Assembled Monolayer on Au(111), Jpn. J. Appl. Phys. 35 (1996) 5866 . 
[61] F.S. Damos, R.C.S. Luz, L.T. Kubota, Determination of Thickness, Dielectric Constant of Thiol Films, and Kinetics of Adsorption Using Surface Plasmon Resonance, Langmuir. 21 (2005) 602-609. https://doi.org/10.1021/la0487038.

[62] I. Zaccari, B.G. Catchpole, S.X. Laurenson, A.G. Davies, C. Wälti, Improving the Dielectric Properties of Ethylene-Glycol Alkanethiol Self-Assembled Monolayers, Langmuir. 30 (2014) 1321-1326. https://doi.org/10.1021/la403983b.

[63] E.B. Bahadir, M.K. Sezgintürk, A comparative study of short chain and long chain mercapto acids used in biosensor fabrication: A VEGF-R1-based immunosensor as a model system, Artif. Cells, Nanomedicine Biotechnol. 44 (2016) 462-470. https://doi.org/10.3109/21691401.2014.962743.

[64] G. Bacher, S. Pal, L. Kanungo, S. Bhand, A label-free silver wire based impedimetric immunosensor for detection of aflatoxin M1 in milk, Sensors Actuators, B Chem. 168 (2012) 223-230. https://doi.org/10.1016/j.snb.2012.04.012.

[65] Y.F. Liu, J.J. Tsai, Y.T. Chin, E.C. Liao, C.C. Wu, G.J. Wang, Detection of allergies using a silver nanoparticle modified nanostructured biosensor, Sensors Actuators, B Chem. 171-172 (2012) 1095-1100. https://doi.org/10.1016/j.snb.2012.06.039.

[66] Y. Huang, M.C. Bell, I.I. Suni, Impedance biosensor for peanut protein Ara h 1, Anal. Chem. 80 (2008) 9157-9161. https://doi.org/10.1021/ac801048g.

[67] A. Ahmad, E. Moore, Electrochemical immunosensor modified with self-assembled monolayer of 11-mercaptoundecanoic acid on gold electrodes for detection of benzo[a]pyrene in water, Analyst. 137 (2012) 5839-5844. https://doi.org/10.1039/C2AN35236B.

[68] D. Stan, C.-M. Mihailescu, R. Iosub, C. Moldovan, M. Savin, I. Baciu, Electrochemical studies of homogeneous self-assembled monolayers versus mixed self-assembled monolayers on gold electrode for "label free" detection of heart fatty acid binding protein, Thin Solid Films. 526 (2012) 143-149. https://doi.org/https://doi.org/10.1016/j.tsf.2012.11.028. 